This item was submitted to Loughborough's Research Repository by the author.

Items in Figshare are protected by copyright, with all rights reserved, unless otherwise indicated.

\title{
Student perspectives on the relationship between a curve and its tangent in the transition from Euclidean Geometry to Analysis
}

PLEASE CITE THE PUBLISHED VERSION

http://www.tandfonline.com/doi/abs/10.1080/14794800801916457

\section{PUBLISHER}

Taylor and Francis ( British Society for Research into Learning Mathematics

\section{VERSION}

AM (Accepted Manuscript)

\section{LICENCE}

CC BY-NC-ND 4.0

\section{REPOSITORY RECORD}

Biza, Irene, Constantinos Christou, and Theodossios Zachariades. 2019. "Student Perspectives on the Relationship Between a Curve and Its Tangent in the Transition from Euclidean Geometry to Analysis". figshare. https://hdl.handle.net/2134/8870. 
This item was submitted to Loughborough's Institutional Repository (https://dspace.lboro.ac.uk/) by the author and is made available under the following Creative Commons Licence conditions.

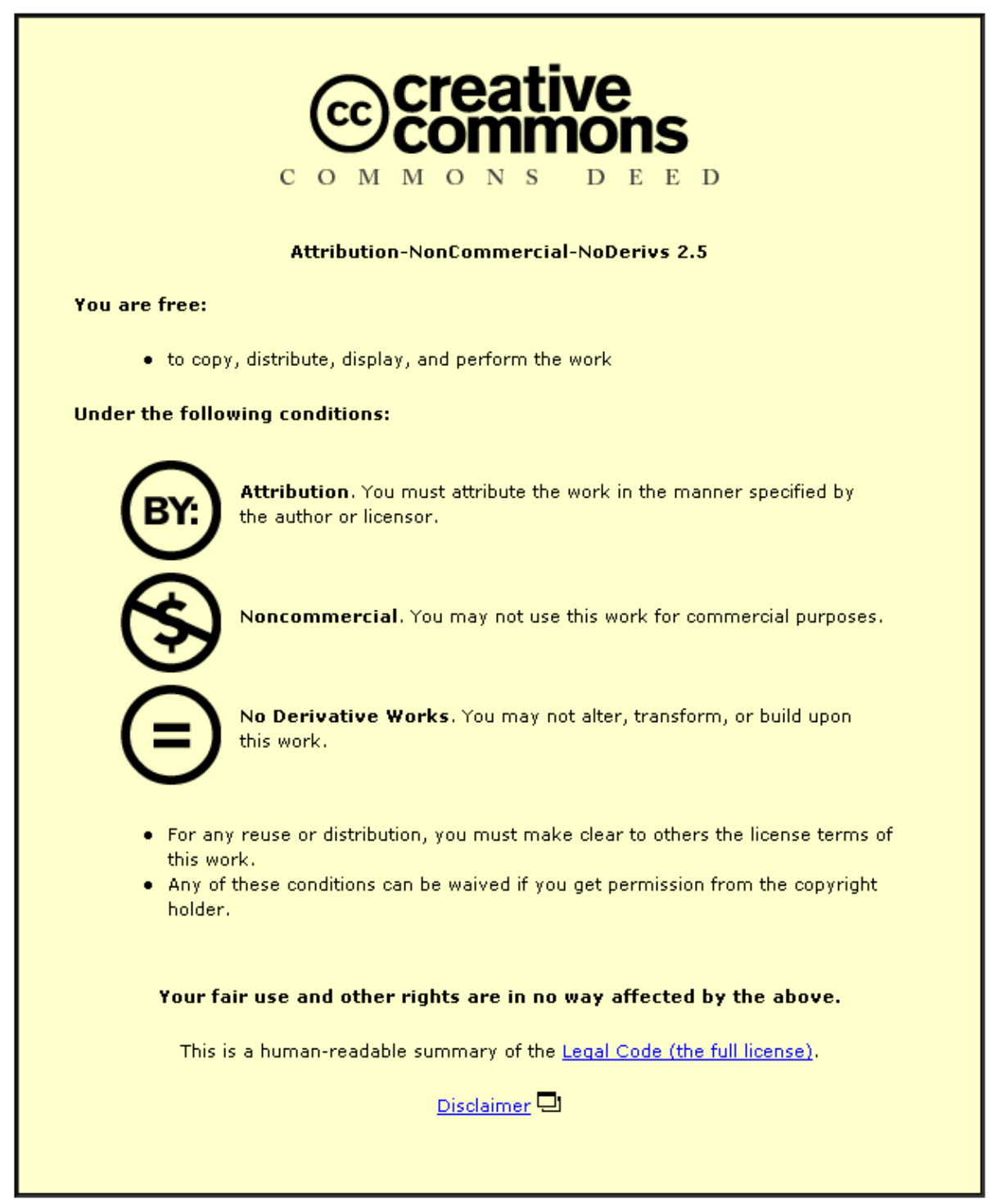

For the full text of this licence, please go to: http://creativecommons.org/licenses/by-nc-nd/2.5/ 


\title{
Student perspectives on the relationship between a curve and its tangent in the transition from Euclidean Geometry to Analysis
}

\author{
Irene Biza*, Constantinos Christou** and Theodossios Zachariades* \\ * Department of Mathematics, University of Athens (Greece) \\ ** Department of Education, University of Cyprus
}

\begin{abstract}
The tangent line is a central concept in many mathematics and science courses. In this paper we describe a model of students' thinking - concept images as well as ability in symbolic manipulation - about the tangent line of a curve as it has developed through students' experiences in Euclidean Geometry and Analysis courses. Data was collected through a questionnaire administered to 196 Year 12 students. Through Latent Class Analysis, the participants were classified in three hierarchical groups representing the transition from a Geometrical Global perspective on the tangent line to an Analytical Local perspective. In the light of this classification, and through qualitative explanations of the students' responses, we describe students' thinking about tangents in terms of seven factors. We confirm the model constituted by these seven factors through Confirmatory Factor Analysis.
\end{abstract}

Keywords: Tangent line, concept image, Analysis

\section{INTRODUCTION}

The concepts of Analysis occupy a central position in the development of mathematical theory. However, they turn out to be subtle and elusive when we try to teach them. Many researchers, especially within the area of Advanced Mathematical Thinking, have 
acknowledged the difficulties in the acquisition of these concepts (e.g. Artigue 1997; Harel, Selden and Selden 2006; Tall 1991a). This paper draws on part of a larger study which focuses on students' understanding of the tangent line, a mathematical concept that is central to many undergraduate mathematics and science topics such as the geometrical interpretation of derivative, the linear approximation of curves, applications in Physics, etc.

Like most concepts in Analysis, the concept of tangent line at a point of a curve is a local notion (e.g. it is defined at a point as the limiting position of secant lines). However, in some cases, such as in that of a tangent to a circle, it has some global characteristics: it keeps the circle in the same semi-plane, for example. Thus, studying the ways in which students think when they work with tangents is important, especially as they meet the concept in different contexts that may require alternating between global and local perspectives. Furthermore, studying this specific concept is important for research on the learning and teaching of Analysis, as it offers a platform for exploring general issues such as the role of definitions in different curricular contexts and educational levels (e.g. Gray et al. 1999; Vinner 1991), the role of representations (e.g. Janvier 1987), and the process of generalisation and abstraction (e.g. Harel and Tall 1991; Tall 1991b).

In this study we investigate how students, who have met the concept of tangent in different contexts, understand the tangent line of a curve at a point. Our purpose in this paper is to describe a model of students' thinking about tangents while they solve relevant problems. To this end, we first provide the theoretical background of our research, then we describe the study and, finally, we present and discuss the results. 


\section{THEORETICAL BACKGROUND}

We consider the structures, which include all the mental pictures, properties and processes associated with a concept, to be students' concept images (Tall and Vinner 1981). We also consider a student's personal (re)construction of the definition of a concept as their personal concept definition. This could be different from the formal concept definition as a form of words used by the mathematical community to specify a mathematical concept (Tall and Vinner 1981).

The tangent line is usually introduced to students in geometry courses in the context of the circle. Later on, students are introduced to a formal or a semi-formal definition of the tangent to a graph of a function. As they build up concept images based on their previous experiences, i.e. on the concept of the tangent of a circle, these concept images may contain coercive elements, such as that a tangent to a more general curve may only meet the curve at one point and may not cross the curve at that point (Vinner 1982, 1991). Tall (1987) defined generic tangent as a line touching the graph at only one point, even where this is inappropriate. In addition, Fischbein (1987) regarded the tangent to a circle as a paradigmatic model of the tangent line. This particular instance of a tangent becomes a tacit theory of what tangents are, and influences the ways in which students think of, and deal with tangents.

The images of the tangent line as described above prove to be inappropriate when the tangent coincides with the graph, or a part of it (Castela 1995; Tall 1987; Vinner 1982, 1991) and, moreover, when specific cases of points are considered, such as inflection points (Castela 1995; Tall 1987; Tsamir and Ovodenko 2004; Vinner 1982, 1991) or points in which the derivative from the left and the right are different real numbers (edge points) where the curve is not smooth (Castela 1995; Tall 1987; Tsamir, Rasslan, and Dreyfus 2006). 
One interesting aspect of the notion of tangent line is that, although the name of the notion remains the same, its definition varies across different curricular contexts. Winicki and Leikin (2000) described how the set of defining conditions of the tangent line, necessary and sufficient properties, interrelates to the set of the corresponding curves. When students - who have studied the tangent line in the case of circle or of specific groups of curves (e.g. conic sections, convex curves or closed and convex curves) - meet the general case of the function graph, they may perceive as defining conditions properties, such as that the tangent has only one common point with the curve, or that the curve is on one side of the tangent line, that are not generally valid (Winicki and Leikin 2000). It appears that, as in the case of several other notions, the acquisition of the general meaning of the tangent follows a trajectory to generalisation that requires quite difficult reconstructions of mathematical knowledge (e.g. Selden 2005).

Harel and Tall (1991) described different kinds of generalisation which depend on the individual's mental construction. They refer to expansive generalisation as one which extends students' existing cognitive structures without requiring changes in their current ideas. They also refer to reconstructive generalisation as one which requires reconstruction of the existing cognitive structure. In reconstructive generalisation, the old concept image has to be changed radically so as to be applicable in a broader context. Expansive and reconstructive generalisations are appropriate for cognitive development and "... although expansive generalization may be easier in the short term, in the long term there are times when the reorganization of knowledge becomes essential, in which reconstructive generalization is far more appropriate" (Harel and Tall 1991, 39-40). 
In the case of the tangent line, Castela (1995) observed that the perspectives that characterise tangency in Euclidean Geometry are the global relations between figures (e.g. the tangent line is a line that has only one common point with the circle), whereas the perspective that characterises tangency in Analysis is local (e.g. the tangent line through point $A$ is the limiting position of secant lines $A B$; is the linear approximation of the curve at this point; has the slope of the curve at this point; etc.). The latter perception is epistemologically different from the former. For the transition from the global (Geometrical) to the local (Analytical) point of view of the tangent line, expansive generalisation, as introduced by Harel and Tall (1991)), could be adequate only for specific groups of curves (e.g. conic sections in Analytic Geometry), whereas, reconstructive generalisation is needed for the transition to the general case of the function graph.

Our research takes into account existing results about the influence of students' initial conceptualisations of the tangent line within a given context (e.g. Euclidean Geometry) on their understanding in subsequent contexts (e.g. Analysis). We contribute to the above results through the creation of a model describing how students, who have met the tangent line in different contexts (Geometrical and Analytical), think about tangents. For this purpose, we first of all investigate statistically the existence of distinct groups of students with regard to their responses to problems about tangents. Then, we interpret the above classification in terms of students' perspectives on tangents, and we look for influential factors that characterise students' thinking. Finally, we propose a model based on the above observations and we confirm this model through statistical methods.

\section{THE STUDY}

In the Greek educational setting, in which this study was conducted, students encounter the notion of tangent line in different contexts. Firstly, they encounter the tangent to the 
circle in Year 10 (aged fifteen to sixteen years) in the context of Euclidean Geometry. Then, in Year 11, they are introduced to the tangent lines of conic sections in the context of Analytic Geometry. Finally, in Year 12, the tangent line to a function graph is defined as a line with slope equal to the derivative of the corresponding function at the point of tangency. Although in Years 11 and 12 the tangent is introduced as the limiting position of secant lines, this definition is rarely used in problems and applications.

The above description suggests that students encounter the concept of tangent in three mathematical contexts, in Euclidean Geometry, in Analytic Geometry and in Analysis, and they experience it in different courses. This led us to believe that the Greek curricular setting offers ample opportunity to study issues concerning the effect that the different contexts in which the tangent line has been experienced has on students' cognitive structures associated with the concept.

Participants in this study were 196 Year 12 students from nine Greek secondary schools. All students had mathematics as a major subject and, by the time the research took place, they had already been taught elementary Calculus, including functions, limit, continuity, derivative, tangent line and the applications of derivative to monotonic functions, extreme values, concavity, inflection points and curve sketching.

\section{The Questionnaire}

Data was collected through a questionnaire (see Figure 1) which was administered to the participants in the middle of the second semester. Students' engagement with the questionnaire lasted approximately one hour and took place during mathematical lessons. The initial design of the questionnaire was based on existing research results concerning tangents as presented in Tall (1987) and Vinner (1982, 1991). The final 
version of the questionnaire was developed after a pilot application with students in Years 12 and first-year mathematics undergraduates (Biza, Souyoul and Zachariades 2005).

[Insert Figure 1 about here]

The questionnaire contained eight questions (q1 to q8), some of which were subdivided. The coding of questions and, what we regarded as correct choices in questions q3, q4, $\mathrm{q} 5, \mathrm{q} 7$, and q8 are presented in columns one and two of Table 2.

In question q3 we asked students to identify whether the drawn lines are tangent lines (identification tasks) and in q4 and q5 to construct a tangent line through a specific point $A$ on the curve or outside the curve (construction tasks), without giving them any formula. Specifically, in question q5 there were three different tangent lines to the curve through point $A$ : one that touches the curve only at the point of tangency, coded q5.1, one that intersects the curve in its extension after the point of tangency, coded q5.2, and one that has to intersect with the curve in order to reach the point of tangency, coded q5.3 (see Table 2). Students were prompted to write their ideas in q1 and q2 and to justify their choices in $\mathrm{q} 3, \mathrm{q} 4$, and $\mathrm{q} 5$, without necessarily using symbolic mathematical language or mathematical terms. Questions q6, q7 and q8 asked students to provide definitions, to write formulae, and to apply formulae in specific cases. These questions were presented in the same way as in the textbooks the students were familiar with.

Questions q1, q2, q6 and q7 (particularly q1 and q6) examined students personal concept definition as opposed to the formal concept definition, and their concept image in general, as derived through their overall responses (Tall and Vinner 1981). Questions q1 and q2 asked for students' spontaneous ideas; q7 referred to the formula, aiming to explore students' recollection and knowledge of the symbolic representation of the 
tangent; and q6 asked for the definition of tangent, expressed verbally and/or

symbolically (and possibly graphically).

\section{Analysis}

The aim of the analysis was to propose a model that describes students' thinking about tangents in terms of factors derived from the data. To this end, we first classified students in distinct groups using Latent Class Analysis (LCA). LCA is a statistical method for finding subtypes of related cases (latent classes) from multivariate categorical data. That is, given a sample of subjects, measured against several variables, one explores whether there is a small number of basic groups into which subjects fall. Through LCA, we examined whether there are different types of students in our sample who could represent different ways of thinking about tangents. LCA was based on students' responses in q3, q4, q5 (only the part of identification or construction of the right line) and in $\mathrm{q} 7$ and $\mathrm{q} 8$. The correct responses were marked as 1 , and both incorrect responses and unanswered questions were marked as 0 .

Subsequently, and taking into consideration the classification of students into groups, we scrutinised students' answers to q1, q2 and q6, and their justifications to q3, q4 and q5, in order to seek some qualitative explanations for students' responses, and, through these, to describe the ways of thinking in each group. Through an examination of similarities within each group as well as variations across groups, we identified influential factors on students' thinking about tangents.

We confirmed the model constituted by these factors through Confirmatory Factor Analysis (CFA). For these analyses we used the structural equation modelling software MPLUSv4.21 (Muthen and Muthen 2007).

In this study, we based our analysis on the students' performance in the questionnaire, and we used written answers and reasoning to make conjectures about students' concept 
images and personal concept definitions. The reasons for choosing this method of analysis were threefold. First, we wanted to have access to a relatively large number of students in order to investigate a broader spectrum of variation on conceptualisations of tangents. Our second aim was the statistical verification of the existence of student groups in relation to difficulties with the tangent concept identified in previous research, and the enrichment of our knowledge about these issues through this grouping. Finally, our purpose was to complement the work of qualitative studies that describe students' thinking about tangents by introducing a model based on influential factors that could be applied in data coding.

\section{RESULTS}

In order to search for different levels in students' abilities to recognise and construct tangents and manipulate them symbolically, we analysed the data using LCA. We decided which model best fit the data by using the following criteria: the smallest Bayesian Information Criterion (BIC); the probabilities that a student belongs to a specific group; and the generation of substantively meaningful groups, with regard to previous theory on students' perceptions of tangent lines, the aims of the study and the students' responses to the entire questionnaire (Muthen, 2001).

Through LCA students were classified into three groups: group A with 78 students, group B with 60 students and group C with 58 students. These three groups are distinctly different, since the probability that a student belongs to the group where s/he was classified in the diagonal numbers of Table 1 is more than 0.9 .

[Insert Table 1 about here]

Table 2 presents the questions of the questionnaire used in LCA: column 1 lists the names of the questions; column 2 the correct answers; column 3 the performance $(\%)$ in 
each question across all students; and, columns 4, 5 and 6 the performance (\%) in each question for each group. Questions were arranged in decreasing order with regard to performance across all students (as in column 3). The numbers above the bold zigzag line relate to the questions that more than $50 \%$ of the students in the corresponding group answered correctly. In this study, we consider a group's performance in a question to be satisfactory when this question is answered correctly by more than $50 \%$ of the students classified in this group (above the zigzag line). We deduce from Table 2 that there is a hierarchy in students' abilities to complete the questionnaire tasks, as success in any question by more than half of the students in a group is associated with success by more than half of the students in all subsequent groups. For example, in Table 2, the students' performance in question $\mathrm{q} 4.3$ is above the zigzag line for group $\mathrm{C}$, indicating that it was answered correctly by at least half of the students in this group (78\%). In the sense of the hierarchy described above, the corresponding performances for groups B and A, in Table 2, will be above the zigzag line as well.

[Insert Table 2 about here]

\section{Different perspectives on the tangent line}

After the classification of students in groups, we scrutinised students' answers, and justifications, in the entire questionnaire. The aim was to build a profile for each group, as well as to identify differences and similarities among groups. In the analysis we present here, the three groups of students represent three basic perspectives on tangents: the Analytical Local, the Intermediate Local, and the Geometrical Global (Table 4). We include below some verbatim quotations from the students' responses to illustrate some of the characteristics within each group, even though a qualitative analysis of the students' responses is beyond the scope of this paper. 


\section{Group A: Analytical Local perspective}

Students belonging to group A demonstrated a satisfactory performance in the questionnaire (all the questions are above the zigzag line as shown in Table 2). The weakest performance of this group is in questions q4.6 and q4.7. Two subgroups of students can be identified in terms of their answers to these questions. The first subgroup of students (65\% of the group) accepted as a tangent the line that coincides with the curve (or a part of it). These students correctly answered at least one of the questions q4.6 and q4.7. The second subgroup seemed not to accept the coincidence of tangent and curve, and thus answered questions q4.6 and q4.7 wrongly.

Another observation concerns the cases in which the point $A$ is an inflection point. Although most students in this group accepted the tangent line at an inflection point, as shown in their performance in questions q3.4 and q4.4, significantly more of them answered q3.4 correctly (94\% rather than 64\%). This difference is observed in all groups (see Table 2). A possible explanation could be students' difficulties in accepting tangents at an inflection point as noted by Castela (1995), Tall (1987) and Vinner (1982, 1991), on particular those in groups B and C. Another reason could be students' problems with identifying the inflection points in a graph, as mentioned by Tsamir and Ovodenko (2004), and consequently with sketching a tangent at those points, even when they are ready to accept a tangent line at an inflection point, in particular those in group A. These difficulties, as well as students' familiarity with the graph of the cubic, seem to affect students' ability to construct the tangent line in $\mathrm{q} 4.4$, although they identified it visually in q3.4. For example, students who accepted the line in q3.4 preferred to sketch another line intending to pass through point $A$ in $\mathrm{q} 4.4$ as shown in Figure 2.

[Insert Figure 2 about here] 
In questions involving symbolic manipulation, students' performance was more than satisfactory ( $96 \%$ in q7 and $87 \%$ in q8). Although, most of the students in this group wrote the right formula for a tangent, less than half of them (32 students) provided an adequate definition of the tangent line in q6. This difference reflects an inconsistency between students' abilities in symbolic manipulation and verbalisation of the concept definition, and exemplifies a certain compartmentalisation of their knowledge (e.g., Vinner 1990; 1991). It is important to note that only four students belonging to this group - amongst all participants - provided a definition of the tangent line as the limiting position of a secant line, or through a chord whose length tends to zero.

In general, we could say that students' choices in this group revealed a sophisticated concept image of the tangent line, involving cases in which the line has more than one common point with the curve, intersects with the curve at an inflection point, could coincide with the curve, and usually in which the tangency point is not an edge point. The students' concept image of the tangent line seems to be built on the local approach to tangency that characterises its use in Analysis, indicating that these students had generally what we call an Analytical Local perspective. This point of view dominated the way that students in this group justified their choices, even in cases where their responses were not adequate, as we exemplify below.

A student, for example, in justifying her answer to q1, connected the tangent line with "lines that cut the curve so that the length of the defined chord tends to zero". The same student in q3.1 sketched points $x_{1}$ and $x_{2}$ on the $x$-axis (see Figure 3), and accepted the line by writing " $A$ : common point, tangency point". In q6, in a consistent way with the argumentation about the limiting process that she used in other questions, she made the sketch shown in Figure 4. 


\section{[Insert Figures 3 and 4 about here]}

Another student identified two different tangent lines, corresponding to the two parts of the curve, on the right and the left side of the tangency point. This led him to identify, in some cases, one tangent line when two lines coincided, or two different lines when they did not. As a result, he answered the questions about inflection point correctly, stating in q3.4 that "There are 2 tangents. Point $A$ could be considered as a tangency point of two different curves. One on the right of $A$, and one on the left". Later, in answering q4.4, he sketched the correct line, justifying his response by specifying that "the tangents are two. The one touches the convex curve and the other the concave curve". However, this way of thinking proves ineffective in providing correct answers to tasks involving edge points (see Figure 5).

[Insert Figure 5 about here]

\section{Group B: Intermediate Local perspective}

Students belonging to group B demonstrated an unsatisfactory performance in questions $\mathrm{q} 3.4, \mathrm{q} 4.4, \mathrm{q} 4.6, \mathrm{q} 4.7$ and $\mathrm{q} 5.3$ (their performance was under the zigzag line as shown in Table 2). The majority of these students rejected the tangents at an inflection point, q3.4 and $\mathrm{q} 4$, and did not accept a tangent line that coincides with the curve, $\mathrm{q} 4.6$ and $\mathrm{q} 4.7$. Additionally, the performance of students in q3.4 (20\%) was much lower than their performance in q8 (70\%), although both questions corresponded to similar functions. It seems that there is an incompatibility between graphical and symbolic manipulation, indicating inconsistencies in students' responses in the case of inflection points.

Concerning the cases in which the tangent line has another common point with the curve, students' performance was more than $82 \%$ in q3.1, q3.2 and q5.2, and less than $50 \%$ only in q5.3. We could say that students in this group accepted the tangent line 
when it has another common point with the curve, although they did not perform satisfactorily in q5.3. We supposed that students in question q5 probably did not draw the line corresponding to q5.3 not because they did not accept it as a tangent line, but because they did not see it.

Furthermore, students in this group performed satisfactorily on the questions involving edge points, q3.3, q3.5, and q4.5. Specifically, students' performance in these tasks was better than the performance of students in group A (see Table 2). Additionally, eight students (13\% of students in group B) failed in at least one of the questions q4.1, q4.2 and q4.3. The most common error was the drawing of more than one tangent line passing through $A$.

It seems that the choices of students in this group revealed a concept image of the tangent line that has the following characteristics: the line could have more than one common point with the curve, but there is a neighbourhood around the tangency point where there is no other common point between the line and the curve; the tangent line cannot intersect with the curve at the tangency point (e.g. at an inflection point); and, there is no tangent line at an edge point. The first two statements substantiate the concept that there is a neighbourhood around the tangency point, in which the curve remains in the same semi-plane of the tangent line.

These students may have elaborated the properties of the circle tangent (e.g. the one common point or the remaining of the curve in the same semi-plane) in order to apply them in a neighbourhood of the tangency point $A$. These elaborated images of the tangent remain inadequate for the general case of the function graph, as they do not include cases such as the tangent line at an inflection point or the coincidence of the tangent and the curve. Students seem to apply locally (in a neighbourhood) a global property without managing to reconstruct their concept images to include an Analytical 
approach. In Harel and Tall's terms (1991), the existing cognitive structure of students' conceptualisation of the tangent to a circle was expanded and not reconstructed in the context of Analysis. This leads us to claim that these students on the whole had what we call an Intermediate Local perspective, as evident, for example, in the following excerpts.

A student in this group, in answering q2, stated that:

The figure and the tangent have only one common point in an interval defined by us; in this interval (if we are talking about a function graph) the graph remains either above or below the tangent line; in each point the tangent line is unique.

Consistently with the above justification, the student defined in questions q3.1 and q3.2 an interval $\left[x_{1}, x_{2}\right]$ in the $x$-axis (see Figure 6) and indicated that "The $\varepsilon$ is a tangent because in the interval $\left[x_{1}, x_{2}\right]$ it has one common point [with the graph] and the graph remains above the line". Later, she rejected the line in question q3.4 and sketched an unacceptable line in q4.4. In q4.6 and q4.7 she did not sketch any line, and justified her answer by stating that "The straight line does not have a tangent".

[Insert Figure 6 about here]

\section{Group C: Geometrical Global perspective}

Participants belonging to group $\mathrm{C}$ demonstrated a satisfactory performance (above the zigzag line) only in the first six questions shown in Table 2, q3.1, q4.1, q4.2, q4.3, q5.1 and q5.2. The students' performance in questions q3.2, q4.7, q4.6 and q5.3 in Table 2 was less than $50 \%$, and fewer students answered q3.1 and q5.2 correctly than students in groups A and B. Thus, the majority of these students rejected as a tangent line a line that has more than one common point with the curve, while some of them probably accepted the line in q3.1 and q5.2 as a tangent line because only the tangency point was visible. 
Most students in this group did not accept the tangent line at an inflection point (only 6 of them answered both q3.4 and q4.4), but, compared with group B, their performance was better. Additionally, for most of the students in group C, the smoothness of the curve at point $A$ was not a presupposition for the existence of a tangent line at this point, since they accepted as tangents the lines at the edge points. Furthermore, students' fluency with symbolic manipulation in this group was not satisfactory, as the performance in q7 and q8 is $48 \%$ and $31 \%$, respectively.

Eleven students (19\% of group C) failed in at least one of the questions q4.1, q4.2 and $\mathrm{q} 4.3$. The most frequent error, as with group B, was drawing more than one tangent line passing through point $A$. Usually this was accompanied, unlike in group $\mathrm{B}$, with the same error in q4.5 (see Figure 7), or the selection of two or all of the lines in q3.3.

[Insert Figure 7 about here]

The response of students in group C was influenced by the circle tangent properties one common point and remaining in one semi-plane - in an elementary and direct way. However, most of them applied one or both of these properties without having in mind the image of the circle as a smooth curve. Contrary to the previous two groups, a larger number of students in group $\mathrm{C}$ made their choices and formed their justifications through looking at properties of the whole graph rather than looking at a neighbourhood of the common point. The latter implies that these students had what we generally call the Geometrical Global perspective that characterises the concept of tangent in Euclidean Geometry.

In summary, students' choices in the questionnaire revealed several concept images about the tangent line. One such concept image is that the tangent line is the one that has only one common point with the curve and leaves the curve in the same semi-plane. 
Consequently, students with this concept image could accept a tangent at an edge point, but did not accept it at an inflection point. For example, a student in answering question q3.2 stated that: "The line $(\varepsilon)$ is not a tangent to the graph at point $A$ because the line $(\varepsilon)$ cuts the graph at another point”. Based on that conception, the same student rejected the line $(\varepsilon)$ in $\mathrm{q} 3.4$, explaining that "... it cuts the curve at $A$ ". Furthermore, in answering q3.3, he accepted $\left(\varepsilon_{1}\right)$ and $\left(\varepsilon_{2}\right)$ and rejected $\left(\varepsilon_{3}\right)$, and in q3.5 he accepted the sketched line. Another student in answering q3.2 pointed out that "It [the line] isn't [a tangent line] because the tangent line should pass outside of the curve and this [the sketched line] cuts [the curve] in two points and this is incorrect for a tangent line" Another concept image appearing in group $\mathrm{C}$ is that the tangent line is the one that has only one common point with the curve, independent of their relative positions. Consequently, students with this concept image could accept a tangent at an inflection point. This could explain why students in group C performed better than students in group B in questions q3.4 and q4.4. The following excerpt from a student answering q3.1 and q3.2 exemplifies this: " $\varepsilon$ is not a tangent line because it cuts the figure at its extension”. In the same way, this student in justifying her answer in question q3.3 indicated that " $\varepsilon_{1}, \varepsilon_{2}$ and $\varepsilon_{3}$ are tangents, because they have only one common point with the function". Moreover, she accepted $(\varepsilon)$ as a tangent line in $\mathrm{q} 3.4$, because "... as far as it could be extended it will not cut the function". More explicitly, in her answer to q1 she wrote:

We call tangent line the line that touches the curve at only one point. Thus, if we extend this line it does not cut the curve at any other point except the tangency one. It has only one common point with the function.

\section{Model describing students' thinking about tangents}


In summary:

- Almost all students demonstrate a satisfactory performance (above the zigzag line in Table 2) in q5.1 in which the tangent line has only one common point with the curve (more than $93 \%$ of students in each group).

- Students' performance in q3.1, q5.2, q3.2, and q5.3 increased from group C to group B. Comparing groups A and B, the performance was almost the same in $\mathrm{q} 3.1, \mathrm{q} 5.2$, and better in group A concerning q3.2, q5.3. In these questions the tangent line and the curve have more than one common point, but there is a neighbourhood around the tangency point where no other common point exists.

- Only group A demonstrated a satisfactory performance in q4.6 and q4.7. In these questions, the tangent line has an infinite number of common points with the curve in any neighbourhood of the tangency point as it coincides with the curve (or part of it). The performance of students belonging to group B is similar to the performance of group C students in $\mathrm{q} 4.7$, while group $\mathrm{C}$ students did better than group B students in question q4.6.

- Only group A demonstrated satisfactory performance in q3.4 and q4.4, in which point $A$ is an inflection point. Furthermore, the level of performance of students belonging to group $\mathrm{C}$ was higher than the performance of students in group B.

- Only group C did not perform satisfactorily in q3.3, q3.5 and q4.5, in which point $A$ is an edge point. The level of performance in these questions increases from group C to group A, and then to group B.

- Only group C did not perform satisfactorily in questions involving symbolic manipulation, q7 and q8. The level of performance in these questions increases from group C to group B and then to group A. 
- All the questions involving conic sections, q4.1, q4.2 and q4.3, were above the zigzag line for all groups, however, students in group A performed better than students in groups B and C.

The above summary highlights several issues regarding students' thinking about tangent lines. These issues relate to specific properties, or aspects, of the tangent line, as addressed by specific questions in the questionnaire. We present these issues as influential factors on the students' thinking about tangents, in Table 3.

[Insert Table 3 about here]

Using Confirmatory Factor Analysis, we confirmed that six of these factors, F2-F7, constitute a model describing students' thinking about tangents. F1 is omitted, as almost all students answered correctly the corresponding question, q5.1. Due to limitations of space, we only provide the confirmation indices of the model: $x^{2} / d f=65.933 / 33=1.99$ (should be less than 2), CFI $=0.961$ (should be more than 0.9), RMSEA $=0.072$ (should be less than 0.08) (for confirmation indices see Marcoulides and Schumacker 1996). By associating the factors shown in Table 3 with the students' performance in the corresponding questions for each group in Table 2, we can deduce that group A performed satisfactorily in terms of all factors; group B in terms of factors F1, F2, F5, F6 and F7; and group C only in terms of factors F1 and F7. In Table 4, for each of the three groups, we present their perspectives on tangents, dominant concept images, and corresponding influential factors. As this table illustrates, if a factor appears for one of the groups, it will appear for all subsequent groups.

[Insert Table 4 about here]

\section{DISCUSSION}


Our results agree with what other studies have revealed about the ways that initial conceptualisations of the tangent line influence students' understanding. The significance of the global or local perspectives (Castela 1995) of the tangent concept is particularly acute when the tangent line has more than one common point or when it coincides with the curve, as well as in cases of inflection and edge points (Castela 1995; Tall 1987; Tsamir and Ovodenko 2004; Tsamir, Rasslan, and Dreyfus 2006; Vinner 1982, 1991).

Through the analysis of our data, we identified a hierarchy of three groups (A, B and C) within a sample of students who have been taught tangents in Euclidean and Analytic Geometry as well as in Analysis. In group C, students approached the tangent as a line with a specific relation to the entire curve. They preserved their global perspective on the tangent they had created in Euclidean Geometry. Thus we claimed that students in this group have a Geometrical Global perspective on the tangent. In contrast, students in group A adopted an Analytical Local perspective on the relation between the curve and its tangent. These students appear to have a reconstructed image about tangents, in the terms of reconstructive and expansive generalisation proposed by Harel and Tall (1991)). Additional to the above, there is an intermediate group of students, group B. The students of this group did not appear to have a reconstructed image about tangents; their image remains based on the global perspective. They tried to assimilate new information about function graph tangent into their existing knowledge in the case of the circle. Therefore, they simply expanded the application of properties that were wellknown to them, such as one common point and remaining in the same semi-plane, in a neighbourhood of the tangency point. We claimed that this group adopted an Intermediate Local perspective. 
The proposed factors presented in Table 3 represent aspects of the tangent line through which we can study the transition from the global to the local perspective. In terms of these factors, students' concept images may be clearly influenced, but very variably, by the circle tangent, and these influences are evident in some new and often incorrect structures. For some of the choices students made we could identify the underlying personal concept definitions and reasoning. For example, in group $\mathrm{C}$ there were students who performed satisfactorily with regard to F1, F4 and F7 but not F5. A possible personal concept definition for them could be: "a line is a tangent line if (and only if) it has one common point with the curve". On the other hand, students in group B who had assimilated the properties of circle tangent in a neighbourhood of the tangency point (satisfactory performance with regard to F1, F2, F5, F7 but not F4) could define the tangent line as "a line that has one common point with the curve in a neighbourhood of tangency point but does not split the curve". We investigate students' personal concept definitions further in Biza, (in preparation), in which we also include qualitative analyses of individual student responses to the questionnaire.

Through this research, we aim to contribute to our understanding of students' perspectives on tangent lines, an important concept in their introduction to Analysis, and thus facilitate their transition to the Analytical Local perspective. In this sense, the contribution of the proposed model is twofold. Firstly, this model could be applied as a tool for the identification, exploration and description of students' thinking about tangents. Secondly, the teaching of the tangent line in an Analysis course could be facilitated through tasks based on the factors constituting the model. 


\section{ACKNOWLEDGEMENTS}

The project is co-funded by the European Social Fund and National Resources (EPEAEK II) PYTHAGORAS.

\section{REFERENCES}

Artigue, M., 1997. Teaching and learning elementary Analysis: what can we learn from didactical research and curriculum evolution? In Proceedings of First Mediterranean Conference on Mathematics, ed. G. Makridis, 207-219. Nicosia, Cyprus.

Biza, E., in preparation. [Working title: Students' personal concept definitions and reasonings about tangency].

Biza, I., A. Sougioul, and Th. Zachariades. 2005. Conceptual change in advanced mathematical thinking. In Proceedings of the Fourth Conference on European Research in Mathematics Education, ed. M. Bosch, 1727-1736. Sant Feliu de Guixols, Spain. Available at: http://ermeweb.free.fr/CERME4/CERME4_WG14.pdf

Castela, C., 1995. Apprendre avec et contre ses connaissances antérieures: un example concret, celui de la tangente. Recherche en Didactique des Mathématiques 15 (1): 7-47.

Fischbein, E., 1987. Intuition in science and mathematics: an educational approach. Dordrecht, The Netherlands: Reidel.

Gray, E., M. Pinto, D. Pitta, and D. Tall. 1999. Knowledge construction and diverging thinking in elementary and advanced mathematics. Educational Studies in Mathematics 38: 111-133.

Harel, G., A. Selden, and J. Selden. 2006. Advanced mathematical thinking. Some PME perspectives. In Handbook of Research on the Psychology of Mathematics 
Education: Past, Present and Future, ed. A. Gutierrez and P. Boero, 147-172. Rotterdam, The Netherlands: Sense Publishers.

Harel, G., and D. Tall. 1991. The general, the abstract, and the generic in advanced mathematics. For the Learning of Mathematics 11(1): 38-42.

Janvier, C. 1987. Problems of representation in the teaching and learning of mathematics. Hillsdale, NJ: Lawrence Erlbaum Associates.

Marcoulides, G. A., and R. E. Schumacker. 1996. Advanced structural equation modeling: issues and techniques. Mahwah, NJ: Lawrence Erlbaum Associates. Muthen, B. 2001. Latent variable mixture modeling. In New Developments and Techniques in Structural Equation Modeling, ed. G. A. Marcoulides and R. E. Schumacher, 1-33. Mahwah, NJ: Lawrence Erlbaum Associates.

Muthen, L. K., and B. O. Muthen. 2007. Mplus User's Guide. Fourth Edition. Los Angeles, CA: Muthen and Muthen.

Selden, A. 2005. New developments and trends in tertiary mathematics education: or, more of the same? International Journal of Mathematical Education in Science and Technology 36(2/3): 131-147.

Tall, D. O. 1987. Constructing the concept image of a tangent. In Proceedings of the Eleventh PME International Conference, ed. J. C. Bergeron, N. Herscovics and C. Kieran, 69-75. Montréal, Canada. ed. 1991a. Advanced mathematical thinking. Dordrecht, The Netherlands: Kluwer Academic Publishers. . 1991b. The psychology of advanced mathematical thinking. In Advanced Mathematical Thinking, ed. D. Tall, 3-21. Dordrecht, The Netherlands: Kluwer Academic Publishers. 
Tall, D., and S. Vinner. 1981. Concept image and concept definition in mathematics with particular reference to limits and continuity. Educational Studies in Mathematics 12: 151-169.

Tsamir, P., and R. Ovodenko. 2004. Prospective teachers' images and definitions: the case of inflection points. In Proceedings of the 28th PME International Conference, ed. M. J. Høines and A. B. Fuglestad, 337-344. Bergen, Norway.

Tsamir, P., S. Rasslan, and T. Dreyfus. 2006. Prospective teachers' reactions to right-orwrong tasks: The case of derivatives of absolute value functions. Journal of Mathematical Behavior 25: 240-251.

Vinner, S. 1982. Conflicts between definitions and intuitions: the case of the tangent. In Proceedings of the Sixth PME International Conference, ed. A. Vermandel, 2428. Antewerp, Belgium.

. 1990. Inconsistencies: their causes and function in learning mathematics. Focus on Learning Problems in Mathematics 12(3/4): 85-98.

- 1991. The role of definitions in the teaching and learning of mathematics. In Advanced Mathematical Thinking, ed. D. Tall, 65-81. Dordrecht, The Netherlands: Kluwer Academic Publishers.

Winicki, G., and R. Leikin. 2000. On equivalent and non-equivalent definition: part 1. For the learning of Mathematics 20(1): 17-21. 
List of Tables and Figures

Table 1: Average group probabilities

\begin{tabular}{|c|c|c|c|}
\hline Group of classification & A & B & $\mathrm{C}$ \\
\hline $\mathrm{A}$ & 0.902 & 0.083 & 0.015 \\
\hline B & 0.038 & 0.926 & 0.035 \\
\hline $\mathrm{C}$ & 0.009 & 0.032 & 0.959 \\
\hline
\end{tabular}


Table 2: Correct answers and students' performance (\%) in each question. The $\mathbf{X}$ indicates that the sketched line is not accepted or that there is not a tangent line at $A$.

\begin{tabular}{|c|c|c|c|c|c|}
\hline \multirow{2}{*}{ Quest. } & \multirow{2}{*}{ Correct answer } & \multicolumn{4}{|c|}{ Performance (\%) } \\
\hline & & All students & Group A (78) & Group B (60 & Group C (58) \\
\hline g5ed & $\checkmark$ & 94 & 94 & 97 & 93 \\
\hline gand & $\checkmark$ & 94 & 99 & 97 & 84 \\
\hline g5 2.2 & $\checkmark$ & 91 & 95 & 98 & 79 \\
\hline gut & $\checkmark$ & 90 & 99 & 83 & 86 \\
\hline 94.2 & $\checkmark$ & 87 & 99 & 82 & 78 \\
\hline 94.3 & $\checkmark$ & 87 & 100 & 80 & 78 \\
\hline 932 & $\checkmark$ & 76 & 95 & 82 & 43 \\
\hline 93.3 & $\mathbf{x}$ & 75 & 91 & 97 & 31 \\
\hline sh. & $y-f\left(x_{0}\right)=f^{\prime}\left(x_{0}\right)\left(x-x_{0}\right)$ & 75 & 96 & 73 & 48 \\
\hline $\mathrm{q} 3.5$ & $\mathbf{X}$ & 72 & 88 & 98 & 24 \\
\hline 98 & $\begin{array}{l}\text { Calculation of } f(2)=3 \text { and } \\
f^{\prime}(2)=0 \text {, application of the } \\
\text { formula, correct equation: } y=3\end{array}$ & 65 & 87 & 70 & 31 \\
\hline 94.5 & $\mathbf{x}$ & 58 & 73 & 85 & 10 \\
\hline g3.4 & $\checkmark$ & 54 & 94 & 20 & 36 \\
\hline g5.3. & $\checkmark$ & 50 & 85 & 47 & 7 \\
\hline g.4.4 & $\checkmark$ & 33 & 64 & 3 & 21 \\
\hline 94.2 & $\checkmark$ & 32 & 56 & 17 & 16 \\
\hline 94.6 & $\checkmark$ & 32 & 53 & 12 & 24 \\
\hline
\end{tabular}


Table 3: Influential factors on students' thinking about tangent lines

\begin{tabular}{|c|l|}
\hline $\begin{array}{c}\text { Influential } \\
\text { Factor }\end{array}$ & \multicolumn{1}{c|}{ Description and related questions } \\
\hline F1 & $\begin{array}{l}\text { The tangent line could have only one common point with the curve } \\
(\mathrm{q} 5.1)\end{array}$ \\
\hline F2 & $\begin{array}{l}\text { The tangent line could have only one common point in a } \\
\text { neighbourhood of the tangency point (q3.1, q3.2, q5.2, q5.3). }\end{array}$ \\
\hline F3 & $\begin{array}{l}\text { In any neighbourhood of the tangency point the tangent line could } \\
\text { have an infinite number of common points with the curve (q4.6, q4.7). }\end{array}$ \\
\hline F4 & There exists a tangent line at an inflection point (q3.4, q4.4). \\
\hline F5 & There is no tangent line at an edge point (q3.3, q3.5, q4.5). \\
\hline F6 & Symbolic manipulation of the tangent line (q7, q8). \\
\hline F7 & Tangent of conic sections (q4.1, q4.2, q4.3). \\
\hline
\end{tabular}


Table 4: Groups of students: perspectives on tangents, dominant concept images, and corresponding influential factors.

\begin{tabular}{|c|c|c|c|}
\hline Group & Perspective & Dominant concept images & Factors \\
\hline A & 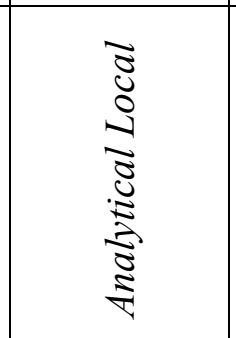 & $\begin{array}{l}\text { The tangent line: } \\
\text { could have more than one common point with the curve; } \\
\text { exists at an inflection point; } \\
\text { could coincide with the curve; and } \\
\text { does not exist at an edge point. } \\
\text { Satisfactory performance in symbolic manipulation. }\end{array}$ & $\begin{array}{c}\text { F1, F2, } \\
\text { F3, F4, } \\
\text { F5, F6, } \\
\text { F7 }\end{array}$ \\
\hline$B$ & 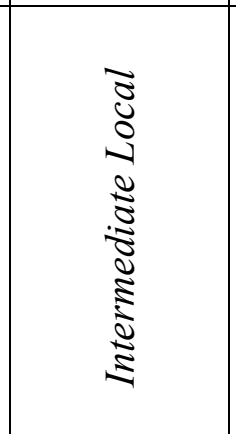 & $\begin{array}{l}\text { The tangent line: } \\
\text { could have more than one common point with the curve } \\
\text { but there is a neighbourhood around the tangency point } \\
\text { where there is no other common point between the line } \\
\text { and the curve; } \\
\text { does not exist at an inflection point; and, } \\
\text { does not exist at an edge point. } \\
\text { Satisfactory performance in symbolic manipulation. }\end{array}$ & $\begin{array}{c}\text { F1, F2, } \\
\text { F5, F6, } \\
\text { F7 }\end{array}$ \\
\hline$C$ & 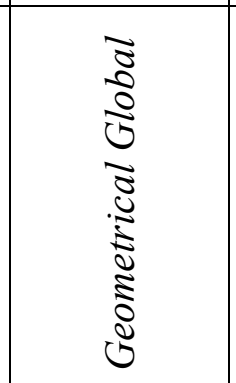 & $\begin{array}{l}\text { The tangent line: } \\
\text { has only one common point with the curve and leaves } \\
\text { the curve in the same semi - plane, or has only one } \\
\text { common point with the curve and could exist at an } \\
\text { inflection point; } \\
\text { could exist at an edge point } \\
\text { Unsatisfactory performance in symbolic manipulation. }\end{array}$ & F1, F7 \\
\hline
\end{tabular}


Figure 1: The questionnaire of the study

Question 1 (q1): Try to explaim, in simple words, what you are thinking when you hear the term "tangent line".

Question 2 (q2): Write as many properties as you can think of about the relationship between a curve and its tangent line at a point $A$.

Question 3 (q3): Which of the lines that are drawn in the following figures are tangent lines of the corresponding graph at point $A$ ? Justify your answers.

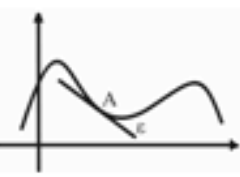

q3.1

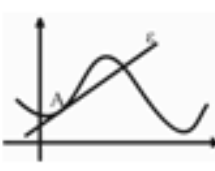

$\mathrm{q} 3.2$

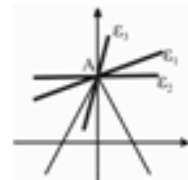

q3.3

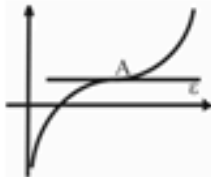

q3.4

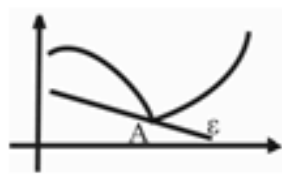

$\mathrm{q} 3.5$

Question 4 (q4): Sketch the tangent lines of the following curves at point $A$, if they exist. Justify your answers.

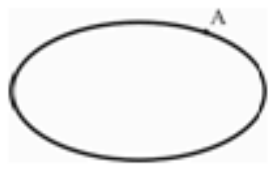

$\mathrm{q} 4.1$

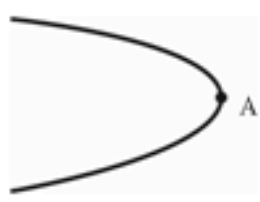

$\mathrm{q} 4.2$

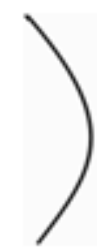

$\mathrm{q} 4.3$

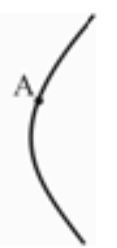

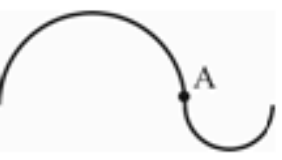

$\mathrm{q} 4.4$

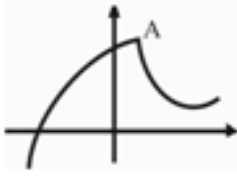

$\mathrm{q} 4.5$

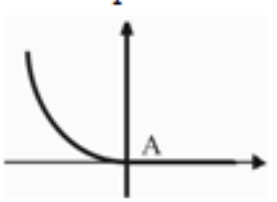

q4.6

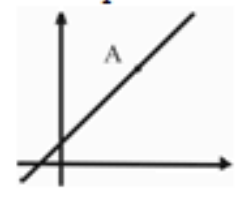

q4.7

Question $5 \mathrm{q}(5)$ : In the following figure, draw as many tangent lines of the curve as you can passing through point $A$.

q5.1, q5.2 and q5.3

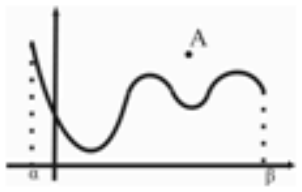

Question 6 (q6): What is the definition of the tangent line of a function graph at its point $A$ ?

Question 7 (q7): Let $f$ be a function and a point $A\left(x_{0}, f\left(x_{0}\right)\right.$ ) of its graph; write the equation of the tangent line of the graph of $f$ at the point $A$, if it exists.

Question 8 (q8): Calculate the equation of the tangent line of the graph of the function $f$ defined by $f(x)=(x-2)^{3}+3$ at the point $A(2, f(2))$. 
Figure 2: Student in group A, question q4.4

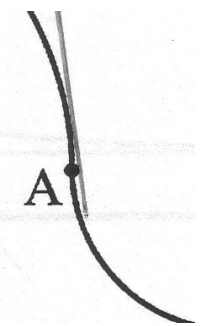

Figure 3: Student in group A, question q3.1

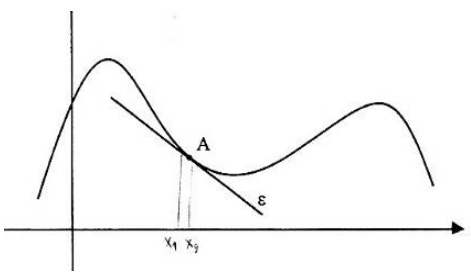

Figure 4: Student in group A, question q6

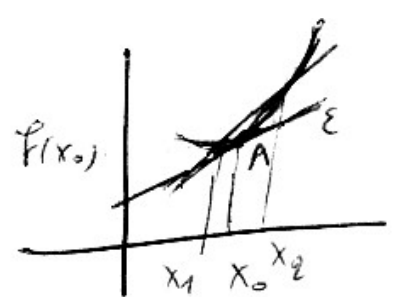

Figure 5: Student in group A, question q4.5

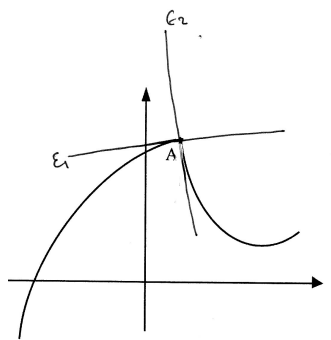

Figure 6: Student in group B, question q3.1

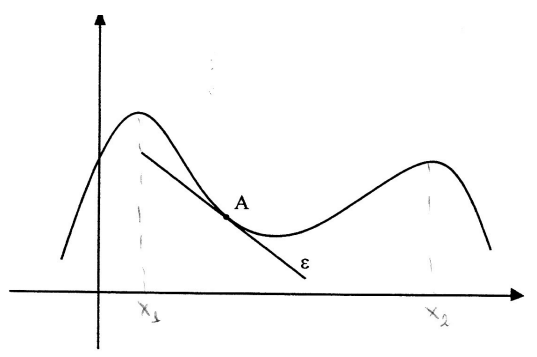

Figure 7: Student in group C, question q4.5

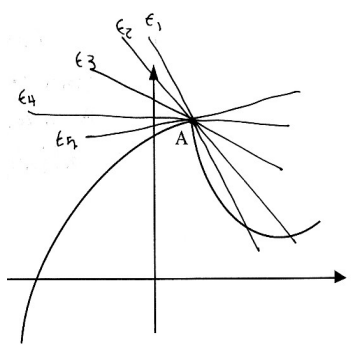

